\title{
Расчет доз аморфизации кремния при облучении легкими ионами средних энергий
}

\author{
(C) Е.В. Окулич ${ }^{1}$, В.И. Окулич ${ }^{2}$, Д.И. Тетельбаум ${ }^{1}$ \\ ${ }^{1}$ Нижегородский государственный университет им. Н.И. Лобачевского, \\ 603950 Нижний Новгород, Россия \\ ${ }^{2}$ Нижегородский институт управления - филиал Российской академии \\ народного хозяйства и государственной службы при Президенте Российской Федерации, \\ 603950 Нижний Новгород, Россия \\ E-mail: eokulich@nifti.unn.ru
}

Поступила в Редакцию 19 декабря 2019 г.

В окончательной редакции 14 января 2020 г.

Принята к публикации 16 апреля 2020 г.

На основе ранее предложенной диффузионно-коагуляционной модели дефектообразования при ионном облучении кремния путем численного решения соответствующих кинетических уравнений рассчитаны дозы аморфизации $\left(\Phi_{\mathrm{am}}\right)$ для ионов средних энергий с массой $M_{1} \leq 31$ а.е.м. Предполагается, что аморфизация на данной глубине происходит при дозе, при которой достигается некоторая пороговая величина суммарной концентрации вакансий и дивакансий $\left(C_{\mathrm{am}}\right)$. Варьируемыми параметрами при расчетах являлись: энергия ионов, плотность ионного тока, температура, а также пороговая энергия смещения атома $\left(E_{d}\right)$ и $C_{\text {am. }}$ Определены границы примененимости диффузионно-коагуляционной модели. Сравнение полученных результатов расчетов, проведенных в этих границах, с опубликованными экспериментальными данными показало (с учетом вариации экспериментальных данных и определенной свободы выбора параметров $E_{d}$ и $\left.C_{\text {am }}\right)$ удовлетворительное соответствие расчетных и экспериментальных значений $\Phi_{\text {am }}$.

Ключевые слова: кремний, облучение легкими ионами, диффузионно-коагуляционная модель дефектообразования, расчет доз аморфизации.

DOI: 10.21883/FTP.2020.08.49649.9338

\section{1. Введение}

Ионно-лучевые методы воздействия на материалы начиная с 70-х годов прошлого века играют весьма важную, а во многих случаях определяющую роль в технологии производства приборов микроэлектронной и наноэлектронной техники. Свойства ионно-облученного полупроводника, как непосредственно после облучения, так и после термической обработки, критическим образом зависят от степени радиационного повреждения, что требует знания зависимости последней от условий облучения. Особенно важна эта проблема для кремния, который был и остается на достаточно длительную перспективу основным полупроводниковым материалом электронной техники.

Особый интерес вызывает явление аморфизации, наблюдающееся при достаточно больших дозах облучения и сильно влияющее на кинетику пострадиационного отжига.

Несмотря на длительную историю исследования аморфизации кремния, большого количества теоретических и экспериментальных работ, посвященных данной проблеме (см., например, [1]), она остается не до конца решенной. Это связано с большим количеством факторов, влияющих на кинетику радиационного повреждения: масса и энергия ионов, температура, плотность ионного тока и доза. Для тяжелых ионов (условно - с атомными массами $M_{1}>40$ а.е.м.) температура при облучении и плотность ионного тока в определенных пределах не оказывают сильного влияния на степень радиационного повреждения. В то же время для легких ионов они являются доминирующими.

Широко используемая для расчета радиационного повреждения общедоступная программа SRIM [2] не учитывает вторичных эффектов, определяющих уровень повреждения во время и после облучения, и потому не может непосредственно применяться для оценки доз аморфизации в случае легких ионов. Метод молекулярной динамики (МД) в принципе позволяет учитывать как первичные, так и вторичные процессы $[3,4]$. Однако ввиду высоких требований к компьютерным ресурсам расчеты этим методом ограничиваются рассмотрением процессов, происходящих в объемах очень малого размера, за времена порядка нескольких наносекунд и менее после прохождения отдельного иона и для объемов малого размера. Однако в $\mathrm{Si}$ вторичные процессы, сопровождающие радиационные повреждения (диффузия, аннигиляция элементарных дефектов - вакансий $(V)$ и собственных межузельных атомов (I), объединение их в более крупные дефекты [5]), при температурах вблизи комнатной происходят за времена, существенно превышающие указанный порядок величины. При этом элементарные дефекты успевают продиффундировать на значительные расстояния, $\sim 10^{-6}-10^{-5} \mathrm{~cm}[6,7]$, что намного больше типичных размеров модельных областей в расчетах методом МД.

В работах [8,9] используется модель аморфизации $\mathrm{Si}$, не требующая учета указанных вторичных процессов 
и опирающаяся на представление о накоплении и рекристаллизации локальных аморфных нанообластей (amorphous pockets, AP), образующихся непосредственно при прохождении иона и представляющих собой, согласно используемой модели $[8,9]$, скопления близко расположенных друг к другу $V$ и $I$ (комплексы вида $\left.I_{m} V_{n}\right)$, координаты которых рассчитываются методом Монте-Карло. В работе [3] наличие локальных разупорядоченных областей с тем же названием АР обнаружено при моделировании методом МД. Однако в [3] констатируется, что для легких ионов (типа бора) AP играют лишь малую роль в разупорядочении по сравнению с одиночными $V$ и $I$. В работе [10] из сравнения экспериментальных данных с модельными оценками для таких ионов, как $\mathrm{Si}^{+}$, также делается вывод о преобладающей (по сравнению с AP) роли разбегающихся из каскадных областей точечных дефектов при комнатной температуре. Таким образом, весьма проблематично использование модели аморфизации, основанной на представлении об образовании и отжиге АР, для ионов с малыми массами.

Трудоемкость расчетов методами МД и динамического метода Монте-Карло, а также сложность интерпретации таких результатов затрудняют оценки дозы аморфизации для широкого набора параметров процесса облучения. В этом отношении преимуществом обладают аналитические методы, основанные на решении кинетических уравнений. В [11] для легких ионов была предложена аналитическая диффузионно-коагуляционная (ДК) модель накопления радиационных дефектов. В [12] на основе этой модели для ионов с атомными массами $M_{1} \leq 31$ а.е.м. были выполнены расчеты с использованием разработанного расчетного комплекса зависимостей степени радиационного повреждения от плотности ионного тока и температуры. При этом, в отличие от [11], были учтены диффузионные процессы.

В настоящей работе приводятся результаты расчетов доз аморфизации кремния легкими ионами $\left(\mathrm{B}^{+}, \mathrm{N}^{+}, \mathrm{Si}^{+}\right.$ и $\mathrm{P}^{+}$) на основе ДК модели в зависимости от плотности ионного тока $(j)$ и температуры мишени $(T)$ при энергиях ионов $E=50$ и 100 кэВ. Результаты расчетов сравниваются с опубликованными экспериментальными литературными данными.

\section{2. Методика расчета}

Прежде всего следует уточнить термин ,доза аморфизации“. Принято представление о том, что аморфизация - это фазовый переход первого рода из кристаллического в разупорядоченное состояние, при котором отсутствует дальний порядок в пространственном распределении атомов [1]. Согласно распространенной модели, впервые предложенной Р. Смолуховским [13] для случая нейтронного облучения и группой исследователей с участием одного из авторов данной работы для ионного облучения [14], аморфизация при ионном облучении происходит в том случае, когда концентрация радиационных дефектов достигает некоторого порогового значения. Так как радиационные дефекты при ионном облучении неоднородно распределены по глубине, логично предположить, что с ростом дозы аморфизация вначале происходит на глубине, где концентрация дефектов максимальна, а затем аморфизованный слой расширяется в обе стороны, т.е. процесс аморфизации растянут по времени и неоднороден по глубине. Если кроме объемного максимума в распределении дефектов возникает приповерхностный максимум $[15,16]$, то формируются две аморфные области, которые при определенной дозе сливаются, образуя единый аморфный слой. Прежде чем образуется аморфный слой (или слои), обычно возникают изолированные аморфные включения [3], и детальная картина аморфизации в общем случае требует трехмерного рассмотрения. Однако в тех случаях, когда интересует доза, при которой происходит сплошная аморфизация на определенной глубине, приемлемо приближенное одномерное рассмотрение (в направлении, перпендикулярном облучаемой поверхности).

В этой связи возникает вопрос об относительности понятия „дозы аморфизации“. С одной стороны, имеющиеся в литературе экспериментальные данные [7,16-22] свидетельствуют об отсутствии систематических исследований влияния параметров процесса облучения на „дозу аморфизации“; с другой стороны, поскольку степень разупорядоченности решетки определялась разными методами, требуется тщательный анализ методики с точки зрения указанных выше особенностей процесса аморфизации.

В данной работе под $\Phi_{\mathrm{am}}$ условно понимается доза, при которой суммарная относительная концентрации вакансий и дивакансий на данной глубине достигает заданной критической величины $\left(C_{\mathrm{am}}\right)$. При этом были заданы три значения $C_{\mathrm{am}}: 2,5$ и $10 \%$ от числа атомов кремния в единице объема $\left(N_{\mathrm{Si}}\right)$. Достижение значения $10 \%$ в точке максимума распределения $C_{\text {am }}$ условно принято за дозу аморфизации облученного слоя, которое обычно приводится в экспериментальных исследованиях.

В соответствии с ДК моделью $[11,12,23]$ рассчитывались усредненные в латеральном направлении концентрации дефектов (вакансий и дивакансий) как функции времени облучения и глубины. При этом не учитывалось формирование тонкого $(\leq 10$ нм $)$ приповерхностного дефектного максимума, механизм образования которого требует отдельного рассмотрения $[15,16]$. При расчетах использовались те же значения параметров, что и в [12]. Здесь приведены результаты расчетов $\Phi_{\text {am }}$ с использованием условия отражающей границы (поверхности) для вакансий. По мнению авторов, это более соответствует реальности, чем условие поглощающей границы. В [12] расчеты концентраций дефектов были нами выполнены также и для случая поглощающей границы. Следует заметить, что выбор вида граничного условия для рассмотренных далее случаев существенным образом не повлиял на концентрацию дефектов на глубинах, близких к глубине расположения максимальной концентрации.

Одним из параметров в ДК модели является значение пороговой энергии смещения атомов кремния $E_{d}$. В литературе $[2,24]$ чаще всего используются два типичных 
Таблица 1. Относительная концентрация имплантированных атомов $f=N_{\mathrm{imp}} / N_{\mathrm{Si}}$ (в \%) на глубине максимума распределения при расчетных значениях $\Phi_{\mathrm{am}}$, полученных в рамках ДК модели

\begin{tabular}{|c|c|c|c|c|c|c|c|c|c|}
\hline \multirow{3}{*}{$\begin{array}{c}j, \\
\mathrm{M \kappa} \mathrm{A} / \mathrm{cm}^{2}\end{array}$} & \multirow{3}{*}{$T, \mathrm{~K}$} & \multicolumn{2}{|c|}{$\mathrm{B}^{+}$} & \multicolumn{2}{|c|}{$\mathrm{N}^{+}$} & \multicolumn{2}{|c|}{$\mathrm{Si}^{+}$} & \multicolumn{2}{|c|}{$\mathrm{P}^{+}$} \\
\hline & & \multicolumn{8}{|c|}{$E$, кэВ } \\
\hline & & 50 & 100 & 50 & 100 & 50 & 100 & 50 & 100 \\
\hline 0.5 & $\begin{array}{l}200 \\
300 \\
400\end{array}$ & $\begin{array}{c}0.30 \\
- \\
-\end{array}$ & $\begin{array}{c}0.30 \\
- \\
-\end{array}$ & $\begin{array}{c}0.10 \\
6.60 \\
-\end{array}$ & $\begin{array}{c}0.10 \\
5.80 \\
-\end{array}$ & $\begin{array}{c}0.03 \\
2.10 \\
11.2 \\
(21.6)\end{array}$ & $\begin{array}{c}0.01 \\
1.10 \\
5.6 \\
(12.1)\end{array}$ & $\begin{array}{c}0.02 \\
1.80 \\
8.4 \\
(18.1)\end{array}$ & $\begin{array}{c}0.01 \\
0.90 \\
4.4 \\
(9.4)\end{array}$ \\
\hline 1 & $\begin{array}{l}200 \\
300 \\
400\end{array}$ & $\begin{array}{c}0.10 \\
7.4 \\
(16.8) \\
-\end{array}$ & $\begin{array}{c}0.10 \\
7.6 \\
(16.3) \\
-\end{array}$ & $\begin{array}{c}0.04 \\
3.30 \\
-\end{array}$ & $\begin{array}{c}0.03 \\
2.90 \\
-\end{array}$ & $\begin{array}{l}0.02 \\
1.10 \\
5.10\end{array}$ & $\begin{array}{l}0.01 \\
0.60 \\
2.80\end{array}$ & $\begin{array}{l}0.02 \\
0.90 \\
4.20\end{array}$ & $\begin{array}{l}0.01 \\
0.50 \\
2.20\end{array}$ \\
\hline 2 & $\begin{array}{l}200 \\
300 \\
400\end{array}$ & $\begin{array}{c}0.05 \\
3.70 \\
-\end{array}$ & $\begin{array}{c}0.05 \\
3.90 \\
-\end{array}$ & $\begin{array}{c}0.03 \\
1.70 \\
8.2 \\
(17.6)\end{array}$ & $\begin{array}{c}0.02 \\
1.50 \\
7.6 \\
(16.3)\end{array}$ & $\begin{array}{l}0.01 \\
0.60 \\
\\
2.60\end{array}$ & $\begin{array}{l}0.01 \\
0.30 \\
1.40\end{array}$ & $\begin{array}{l}0.01 \\
0.50 \\
2.10\end{array}$ & $\begin{array}{l}0.01 \\
0.20 \\
1.10\end{array}$ \\
\hline 5 & $\begin{array}{l}200 \\
300 \\
400\end{array}$ & $\begin{array}{c}0.04 \\
1.60 \\
7.5 \\
(16.1)\end{array}$ & $\begin{array}{c}0.03 \\
1.60 \\
8.1 \\
(17.1)\end{array}$ & $\begin{array}{l}0.02 \\
0.70 \\
3.30\end{array}$ & $\begin{array}{l}0.02 \\
0.60 \\
\\
3.10\end{array}$ & $\begin{array}{l}0.01 \\
0.30 \\
1.00\end{array}$ & $\begin{array}{l}0.01 \\
0.10 \\
0.60\end{array}$ & $\begin{array}{l}0.01 \\
0.20 \\
0.90\end{array}$ & $\begin{array}{l}0.01 \\
0.10 \\
0.50\end{array}$ \\
\hline 10 & $\begin{array}{l}200 \\
300 \\
400\end{array}$ & $\begin{array}{l}0.03 \\
0.80 \\
3.80\end{array}$ & $\begin{array}{l}0.03 \\
0.90 \\
4.10\end{array}$ & $\begin{array}{l}0.02 \\
0.40 \\
1.70\end{array}$ & $\begin{array}{l}0.02 \\
0.40 \\
1.60\end{array}$ & $\begin{array}{l}0.01 \\
0.10 \\
0.50\end{array}$ & $\begin{array}{l}0.01 \\
0.08 \\
0.30\end{array}$ & $\begin{array}{l}0.01 \\
0.10 \\
0.50\end{array}$ & $\begin{array}{l}0.01 \\
0.07 \\
0.20\end{array}$ \\
\hline
\end{tabular}

Примечание. Приведены значения относительных концентраций, рассчитанные при $E_{d}=15$ эВ. Для оценки степени применимости модели для случаев, когда при $E_{d}=15$ эВ относительные концентрации близки к $10 \%$, в скобках приведены значения при $E_{d}=22$ эВ для того же условия.

значения этой величины - 15 и 22 эВ. Эти значения и были использованы в расчетах.

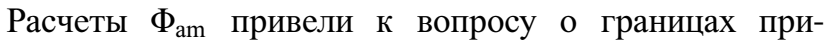
менимости ДК модели. Оказалось, что для некоторых режимов имплантации при расчетной дозе аморфизации концентрация внедряемых ионов оказывается столь большой, что она приближается к $N_{\mathrm{Si}}$. Очевидно, что в этих случаях модель теряет силу хотя бы вследствие влияния примеси на используемые параметры, необходимости учета кластеризации примеси и пр. Очевидно, что ограничение должно быть тем жестче, чем выше химическая активность внедряемых ионов и различие атомных радиусов кремния и примеси. Вопрос о верхнем пределе доз, при котором данный фактор становится существенным, выходит за рамки модели ДК и в общем случае требует применения таких методов моделирования, как молекулярная динамика. В отсутствие точных критериев резонно в первом приближении принять в качестве верхнего предела применимости ДК модели ту дозу, при которой концентрация примеси в максимуме ее распределения $\left(N_{\mathrm{imp}}^{\max }\right)$ мала по сравнению с $N_{\mathrm{Si}}$. В настоящей работе за критическое значение $N_{\mathrm{imp}}^{\max }$ принято значение, равное $0.1 N_{\mathrm{Si}}[25]$. В связи с этим расчетные значения $\Phi_{\mathrm{am}}$, полученные для различных условий облучения, должны быть протестированы на выполнение данного условия, что было реализовано нами с использованием распределениий примесей в объеме $\mathrm{Si}$, полученных с помощью программы SRIM. Далее, в разд. 3, приведены сведения, позволяющие судить о выполнении указанного условия для конкретных значений $j$ и $T$, при двух энергиях, 50 и 100 кэВ, ионов $\mathrm{B}^{+}, \mathrm{N}^{+}, \mathrm{Si}^{+}$и $\mathrm{P}^{+}$. Приведенные далее данные по $\Phi_{\mathrm{am}}$ отвечают критерию $N_{\text {imp }}^{\max }<0.1 N_{\mathrm{Si}}$.

\section{3. Результаты}

Использование описанного в [12] расчетного комплекса позволило достаточно эффективно определить границы применимости ДК модели и получить зависимости $\Phi_{\mathrm{am}}$ в широком диапазоне параметров процесса облучения для указанного выше набора ионов.

В табл. 1 приведены значения $f=N_{\mathrm{imp}} / N_{\mathrm{Si}}$ - относительной концентрации имплантированных атомов в области максимума их распределения (в процентах от $\left.N_{\mathrm{Si}}\right)$, соответствующие расчетным значениям $\Phi_{\mathrm{am}}$ в выражении относительно $N_{\mathrm{Si}}$, в зависимости от условий облучения. Знак „минус“ обозначает факт значительного превышения (более чем в 2 раза) критерия $f<10 \%$.

На рис. 1 приведены расчетные значения $\Phi_{\mathrm{am}}$ на глубине расположения максимума скорости генерации 


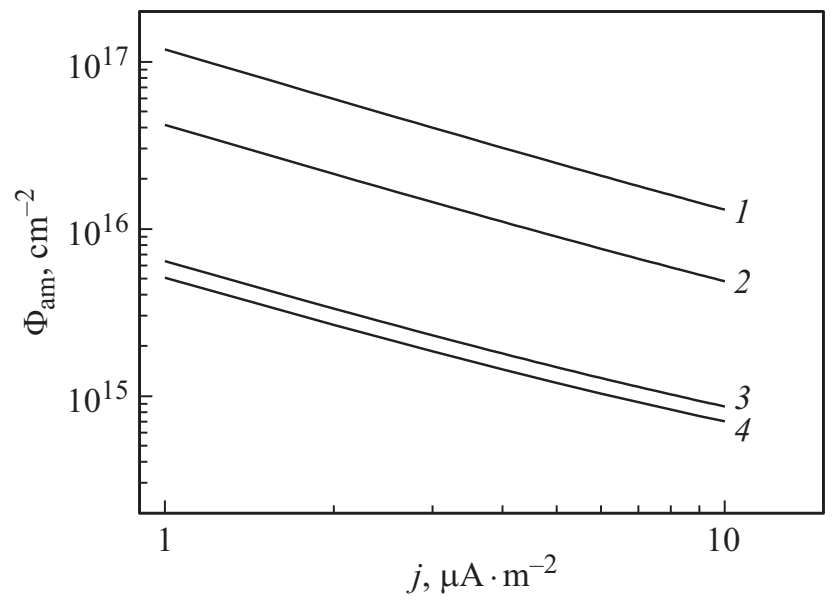

Рис. 1. Зависимость дозы аморфизации в максимуме функции $G(x)$ от плотности ионного тока при $T=300 \mathrm{~K}$ для ионов $\mathrm{B}^{+}(1), \mathrm{N}^{+}(2), \mathrm{Si}^{+}(3), \mathrm{P}^{+}(4) . E=100$ кэВ, $E_{d}=15$ эВ, $C_{\mathrm{am}}=10 \%$.

первичных дефектов $G(x)$ при $E=100$ кэВ и $T=300 \mathrm{~K}$ для нескольких (часто используемых) видов ионов в зависимости от $j$ (ионы во всех случаях считались однозарядными). Параметры процессов вторичного дефектообразования, необходимые для расчетов концентраций вакансий и дивакансий по ДК модели, приведены в [12]. Видно, что как возрастание $j$, так и увеличение массы иона $\left(M_{1}\right)$ приводят к уменьшению $\Phi_{\mathrm{am}}$. Уменьшение дозы аморфизации с увеличением $M_{1}$ при прочих равных условиях связано с увеличением числа дефектов, создаваемых одним ионом, и соответствующим ростом функции $G(x)$. С ростом $M_{1}$ при одной и той же начальной энергии иона увеличивается ее доля, расходуемая на упругие столкновения, что вызывает возрастание функции генерации вакансий $G(x)$ в соответствующих уравнениях [12]. Уменьшение же $\Phi_{\mathrm{am}}$ с ростом $G(x)$ обусловлено тем, что вероятность связывания вакансий в дивакансии возрастает пропорционально квадрату их мгновенной концентрации, тогда как вероятность их захвата ловушками (приводящего, согласно ДК модели, к потере вакансий) пропорциональна первой степени этой величины.

С ростом дозы происходит расширение аморфизованного слоя как вглубь, так и в сторону поверхности. На рис. 2 приведены отношения расчетных доз аморфизации для глубины $h=10 \mathrm{Hм}, \Phi_{\mathrm{am}}(h=10 \mathrm{Hм})$, и для глубины, где расположен максимум функции $G(x), \Phi_{\mathrm{am}}(\max )$, в зависимости от $M_{1}$ при энергииях ионов 50 и 100 кэВ $\left(j=1 \mathrm{м \kappa А} / \mathrm{cm}^{2}\right)$. С увеличением $M_{1}$ отношение приближается к единице. Это обусловлено тем, что с ростом $M_{1}$ уменьшается острота максимума функции $G(x)$ [2]. Интересно, что это отношение практически не зависит от плотности ионного тока в рассмотренном интервале $j$.

На рис. 3 приведены результаты расчета зависимости $\Phi_{\text {am }}$ на глубине расположения максимума суммарных концентраций дефектов (вакансий и дивакансий) для ионов $\mathrm{Si}^{+}$с $E=100$ кэВ от температуры при трех значениях $j$. Величина $\Phi_{\text {am }}$ при высоких $T$ растет с увеличением температуры, а при понижении температуры асимптотически стремится к постоянному значению. Последнее связано с тем, что при низких температурах потеря вакансий за счет их захвата на ловушки уменьшается из-за наличия энергетического барьера для захвата, так что в пределе низких температур вклад в разупорядочение вносят все генерированные при облучении вакансии.

Чтобы дать наглядное представление о чувствительности $\Phi_{\text {am }}$ к различным факторам, на рис. 4 в качестве типичного примера представлены зависимости $\Phi_{\text {am }}$ на глубине максимума функции $G(x)$ для ионов $\mathrm{Si}^{+}$с

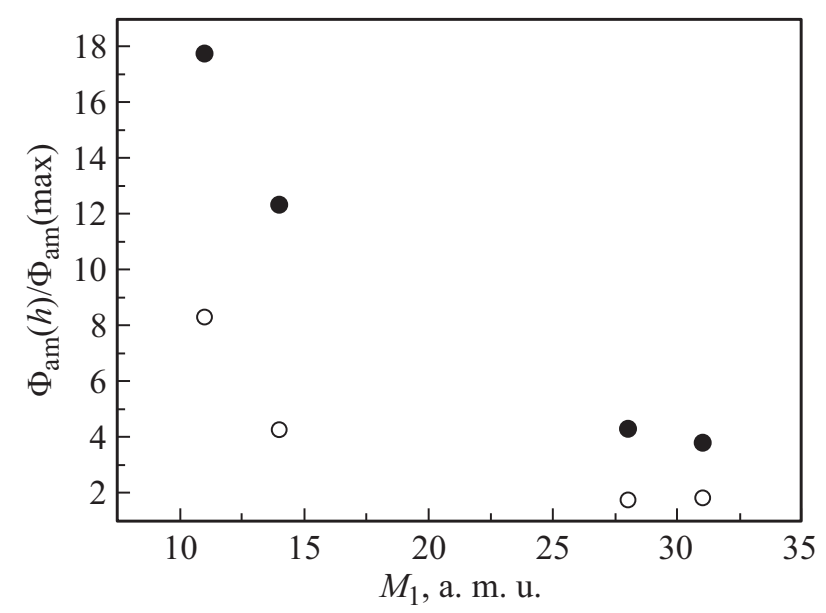

Рис. 2. Зависимость отношения дозы аморфизации на глубине $h=10$ нм к дозе аморфизации на глубине, соответствующей максимуму функции $G(x)$, при энергиях 50 (1) и 100 кэВ (2) от массы иона $M_{1}$ при температуре $300 \mathrm{~K}$.

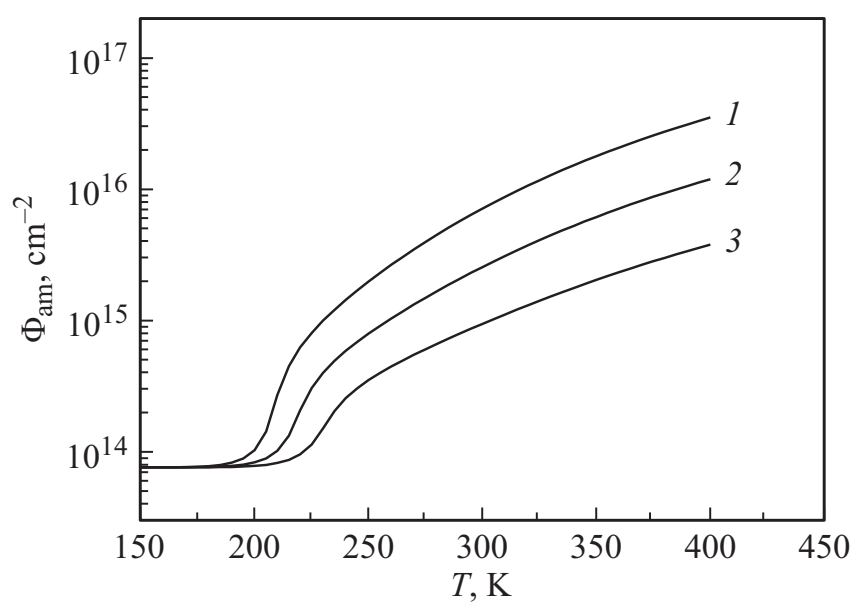

Рис. 3. Зависимость дозы аморфизации на глубине, соответствующей максимуму функции $G(x)$, от температуры для ионов $\mathrm{Si}^{+}\left(E=100\right.$ кэВ) при $j=1(1), 3(2)$, и $10 \mathrm{мкA/ \textrm {cm } ^ { 2 }}$ (3) и $C_{\text {am }}=10 \%$. 
Таблица 2. Зависимость дозы аморфизации от массы и энергии ионов для двух значений температуры облучения

\begin{tabular}{|c|c|c|c|c|c|c|c|}
\hline \multirow[t]{2}{*}{$E_{d}$, эВ } & \multirow{2}{*}{$\begin{array}{c}j, \\
\mathrm{MK} \mathrm{A} / \mathrm{cm}^{2}\end{array}$} & \multirow[t]{2}{*}{$C_{\mathrm{am}}, \%$} & \multirow[t]{2}{*}{$T, \mathrm{~K}$} & \multirow[t]{2}{*}{$E$, кэВ } & \multicolumn{3}{|c|}{$\begin{array}{c}\text { Доза аморфизации, } \\
10^{15} \mathrm{~cm}^{-2}\end{array}$} \\
\hline & & & & & $\mathrm{B}^{+}$ & $\mathrm{N}^{+}$ & $\mathrm{P}^{+}$ \\
\hline \multirow{2}{*}{17} & \multirow{2}{*}{3} & \multirow{2}{*}{5} & 300 & $\begin{array}{r}50 \\
100\end{array}$ & $\begin{array}{c}9.8 \\
14\end{array}$ & $\begin{array}{l}3.9 \\
4.9\end{array}$ & $\begin{array}{l}0.76 \\
0.63\end{array}$ \\
\hline & & & 400 & $\begin{array}{r}50 \\
100\end{array}$ & $\begin{array}{l}49 \\
71\end{array}$ & $\begin{array}{l}19 \\
25\end{array}$ & $\begin{array}{l}3.4 \\
2.9\end{array}$ \\
\hline
\end{tabular}

$E=100$ кэВ от температуры при различных значениях параметров $j, C_{\text {am }}$ и $E_{d}$.

В табл. 2 приведены результаты расчета значений $\Phi_{\text {ат }}$ на глубине максимума функции $G(x)$ для $\mathrm{B}^{+}, \mathrm{N}^{+}$и $\mathrm{P}^{+}$при $j=3$ мкА $/ \mathrm{cm}^{2}, T=300$ и $400 \mathrm{~K}, C_{\text {ат }}=5 \%$. При этом использовалось промежуточное (между 15 и 22 эВ) значение пороговой энергии смещения $E_{d}=17$ эВ.

Сравним теперь результаты с литературными данными. В литературе опубликованы достаточно многочисленные данные по $\Phi_{\mathrm{am}}$. К сожалению, авторы не всегда указывают такой важный параметр, как плотность ионного тока. Не везде гарантируется и отсутствие существенного нагрева образцов ионным пучком. Кроме того, как уже говорилось, нужно иметь в виду, что именно понимается под „дозой аморфизации“ в различных работах. В одних случаях (например, в методе ЭПР) для ионов средних энергий фиксируется переход в аморфное состояние практически всего имплантированного слоя, в другом (метод обратного резерфордовского рассеяния) определяется разупорядочение на разных глубинах; при этом доза, при которой происходит полная аморфизация на глубине расположения максимума профиля распределения степени разупорядочения, определяется с большой погрешностью вследствие замедления роста количества обратно рассеянных ионов с дозой по мере приближения этого количества к уровню, найденному для произвольно разупорядоченного образца. Просвечивающая электронная микроскопия поперечного среза позволяет четко определить наличие и границы аморфного слоя, но этот метод, ввиду его трудоемкости, чаще всего используется лишь для некоторой определенной совокупности параметров облучения $\Phi, j$ и $T$. При сравнении результатов с экспериментальными данными следует иметь в виду также то, что имеется определенная свобода в выборе используемых в расчетах по ДК модели параметров, входящих в уравнения [12].

Сравнение проведено для ионов $\mathrm{B}^{+}, \mathrm{N}^{+}, \mathrm{Si}^{+}, \mathrm{P}^{+}$при двух значениях параметров $E_{d}(15$ и 22 эВ $[2,24])$ и двух значениях критической концентрации дефектов $\left(C_{\mathrm{am}}=5\right.$ и $10 \%)$.

В табл. 3 приведены расчетные значения $\Phi_{\text {am при }}$ комнатной температуре облучения на глубине, соответствующей максимуму функции $G(x)$. Там же приведены экспериментальные данные. С учетом указанных выше обстоятельств соответствие расчетных и экспериментальных данных в целом можно считать удовлетворительным.

Таблица 3. Сравнение расчетных $\left(\Phi_{\mathrm{am}}^{\mathrm{calc}}\right)$ и экспериментальных $\left(\Phi_{\mathrm{am}}^{\mathrm{exp}}\right)$ значений доз аморфизации при комнатной температуре

\begin{tabular}{|c|c|c|c|c|c|c|}
\hline \multirow[b]{2}{*}{ Ион } & \multirow{2}{*}{$\begin{array}{c}E, \\
\text { кэВ }\end{array}$} & \multirow{2}{*}{$\begin{array}{c}j \\
\mathrm{MK} / \mathrm{cm}^{2}\end{array}$} & \multirow{2}{*}{$\begin{array}{c}\Phi_{\mathrm{am}}^{\exp } \\
10^{15} \mathrm{~cm}^{-2}\end{array}$} & \multirow{2}{*}{$\begin{array}{c}\text { Метод } \\
\text { определения }\end{array}$} & \multicolumn{2}{|c|}{$\Phi_{\mathrm{am}}^{\mathrm{calc}}, 10^{15} \mathrm{~cm}^{-2}$} \\
\hline & & & & & $\begin{array}{l}E_{d}=22{ }^{\jmath} \mathrm{B} \\
C_{\mathrm{am}}=10 \%\end{array}$ & $\begin{array}{c}E_{d}=15 \text { эВ } \\
C_{\mathrm{am}}=5 \%\end{array}$ \\
\hline $\mathrm{B}^{+}$ & $\begin{array}{l}50 \\
50 \\
50\end{array}$ & $\begin{array}{c}3 \\
10 \\
2\end{array}$ & $\begin{array}{c}30[17] \\
10[17] \\
20[7]\end{array}$ & $\begin{array}{l}\text { RED } \\
\text { ED }\end{array}$ & $\begin{array}{c}33 \\
10 \\
43.5\end{array}$ & $\begin{array}{l}7.7 \\
2.5 \\
8.2\end{array}$ \\
\hline $\mathrm{N}^{+}$ & $\begin{array}{l}40 \\
50\end{array}$ & $\begin{array}{c}7.6 \\
2\end{array}$ & $\begin{array}{c}1.5[16] \\
4[7]\end{array}$ & $\begin{array}{l}\text { RBS } \\
\text { ED }\end{array}$ & $\begin{array}{c}4.9 \\
17.2\end{array}$ & $\begin{array}{l}1.2 \\
3.2\end{array}$ \\
\hline $\mathrm{Si}^{+}$ & $\begin{array}{r}50 \\
230\end{array}$ & $\begin{array}{c}10 \\
\sim 0.2\end{array}$ & $\begin{array}{c}2.0[18] \\
0.5-0.8 \\
{[19]}\end{array}$ & $\begin{array}{c}\text { ESR } \\
\text { XRD, } \\
\text { RBS }\end{array}$ & $\begin{array}{l}1.0 \\
4.1\end{array}$ & $\begin{array}{c}0.24 \\
1.0\end{array}$ \\
\hline $\mathrm{P}^{+}$ & $\begin{array}{r}50 \\
50 \\
100 \\
200\end{array}$ & $\begin{array}{c}10 \\
2 \\
1 \\
10\end{array}$ & $\begin{array}{c}2.0[20] \\
0.4[7] \\
0.8[21] \\
1.0[22]\end{array}$ & $\begin{array}{c}\text { RBS } \\
\text { ED } \\
\text { RS, } \\
\text { RBS } \\
\text { RBS }\end{array}$ & $\begin{array}{l}0.9 \\
4.3 \\
\\
5.1 \\
0.8\end{array}$ & $\begin{array}{c}0.23 \\
0.8 \\
\\
2.4 \\
0.21\end{array}$ \\
\hline
\end{tabular}

Примечание. RBS - обратное резерфордовское рассеяние, RS - рамановская спектроскопия, ESR - электронный парамагнитный резонанс, $\mathrm{XRD}$ - рентгеновская дифракция, RED — электронография с последовательным удалением слоев, ED - электронография. Рассчитанные значения $\Phi_{\mathrm{am}}^{\mathrm{calc}}$ относятся к области максимума функции $G(x)$. 

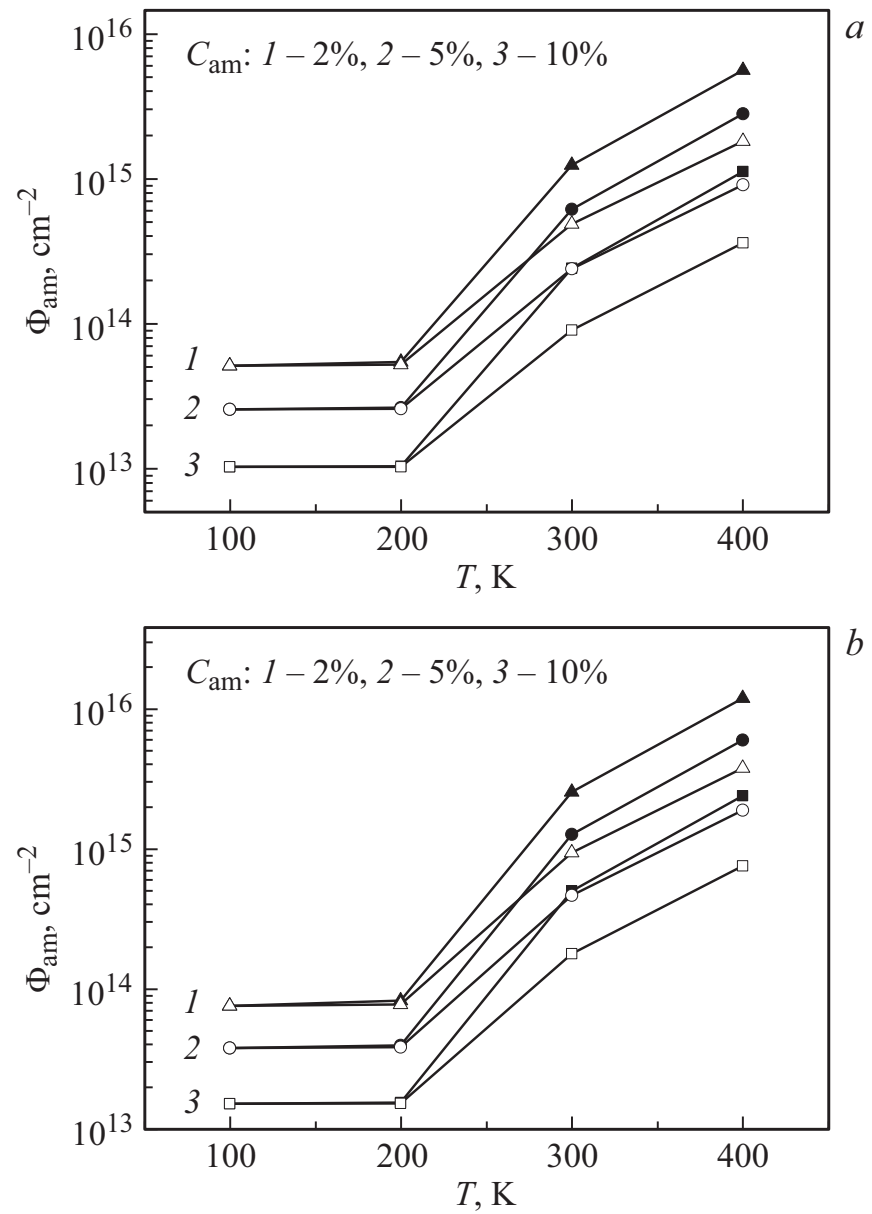

Рис. 4. Доза аморфизации на глубине, соответствующей максимуму функции $G(x)$, при облучении ионами $\mathrm{Si}^{+}$ $\left(E=100\right.$ кэВ) для $j=3$ (темные точки) и $10 \mathrm{M \kappa A} / \mathrm{cm}^{2}$ (светлые точки). $E_{d}=15$ (a) и 22 эВ $(b)$.

Что касается основных предсказываемых закономерностей - зависимости дозы аморфизации от массы иона, плотности ионного тока и температуры облучения - качественно они также удовлетворительно согласуются с экспериментально установленными закономерностями.

\section{4. Заключение}

В работе показано, что ДК модель дает в первом приближении согласующиеся с экспериментальными данными результаты по дозам аморфизации кремния легкими ионами, наиболее часто используемыми в технологии ионной имплантации.

Уточнение результатов в рамках данной модели при использовании разработанного вычислительного комплекса (без привлечения больших компьютерных ресурсов) возможно по мере получения более достоверных сведений о кинетических и других параметрах, входящих в уравнения. С другой стороны, для более точного сравнения с результатами экспериментов в ряде случаев желательна постановка дополнительных опытов при строгой фиксации параметров облучения $(j, T)$, сопровождающихся послойным анализом структурного качества, в частности в области критических доз.

\section{Конфликт интересов}

Авторы заявляют, что у них нет конфликта интересов.

\section{Список литературы}

[1] L. Pelaz, L.A. Marques, J. Barbolla. J. Appl. Phys., 96 (11), 5947 (2004).

[2] J.F. Ziegler, M.D. Ziegler. J.P. Nucl. Instr. Meth. Phys. Res. B, 268 (11-12), 1818 (2010).

[3] M.J. Caturla, T. Diaz de la Rubia, L.A. Marques, G.H. Gilmer. Phys. Rev. B, 54, 16683 (1996).

[4] K. Nordlund. Comput. Mater. Sci., 3, 448 (1995).

[5] В.Л. Винецкий, Г.А. Холодарь. Радиационная физика полупроводников (Киев, Наук. думка, 1979).

[6] А.И. Титов, А.Ю. Азаров, В.С. Беляков. ФТП, 37 (3), 358 (2003).

[7] A.I. Gerasimov, E.I. Zorin, P.V. Pavlov, D.I. Tetelbaum. Phys. Status Solidi A, 12, 679 (1972).

[8] K.R.C. Mok, M. Jaraiz, I. Martin-Bragado, J.E. Rubio, P. Castrillo, R. Pinacho, J. Barbolla, M.P. Srinivasan. J. Appl. Phys., 98, 046104 (2005).

[9] K.R.C. Mok, F. Benistant, M. Jaraiz, J.E. Rubio, P. Castrillo, R. Pinacho, M.P. Srinivasan. J. Appl. Phys., 103, 014911 (2008).

[10] P.D. Edmondson, D.J. Riley, R.C. Birtcher, S.E. Donnelly. J. Appl. Phys., 106, 043505 (2009).

[11] Н.П. Морозов, Д.И. Тетельбаум, П.В. Павлов, Е.И. Зорин. ФТП, 9 (12), 2292 (1975).

[12] Е.В. Окулич, В.И. Окулич, Д.И. Тетельбаум. ФТП, 52 (9), 967 (2018)

[13] Р. Смолуховский. В сб.: Влияние ядерных излучений на материалы, под ред. Дж. Хэрвуда (Л., Судпромгиз, 1961) c. 168

[14] П.В. Павлов, Д.И. Тетельбаум, Е.И. Зорин, Р.В. Кудрявцева. Кристаллография, 12 (1), 155 (1967).

[15] Е.И. Зорин, П.В. Павлов, Д.И. Тетельбаум. Ионное легирование полупроводников (М., Энергия, 1975).

[16] A.I. Titov, G. Carter. Nucl. Instr. Meth. Phys. Res. B, 119, 491 (1996).

[17] Д.И. Тетельбаум. Автореф. докт. дис. (Горький, ГГУ, 1987).

[18] J.G. de Walt, C.A.J. Ammerlau. 2nd Int. Conf. Ion Implantation (N. Y., Springer, 1971) p. 39.

[19] G. Bai, M.A. Nicollet. J. Appl. Phys., 70, 649 (1991).

[20] J.W. Mayer, L. Ericksson, J.A. Davies. Ion Implantation in Semiconductors, Silicon, and Germanium (N.Y., Academic Press, 1970).

[21] T. Motooka, O.W. Holland. Appl. Phys. Lett., 61 (25), (1992).

[22] P.P. Morehed, B.L. Crowder. Rad. Eff., 6, 27 (1970).

[23] N.P. Morozov, D.I. Tetelbaum. Phys. Status Solidi A, 51 (2), 629 (1979)

[24] E. Holmström, A. Kuronen, K. Nordlund. Phys. Rev. B, 78, 045202 (2008).

[25] L.A. Christel, J.F. Gibbons, T.W. Sigmon. J. Appl.Phys., 52, 7143 (1981).

Редактор Л.В. Шаронова 
Calculation of silicon amorphization doses at irradiation with light ions of medium energy

\author{
E.V. Okulich ${ }^{1}$, V.I. Okulich ${ }^{2}$, D.I. Tetelbaum ${ }^{1}$ \\ ${ }^{1}$ Lobachevsky State University of Nizhny Novgorod, \\ 603950 Nizhny Novgorod, Russia \\ ${ }^{2}$ Nizhny Novgorod Institute of Management-Branch \\ of Russian Presidential Academy \\ of National Economy and Public Administration, \\ 603950 Nizhny Novgorod, Russia
}

\begin{abstract}
Based on the previously proposed diffusion-coagulation model of defect formation under ion irradiation of silicon and numerically solving the corresponding kinetic equations, amorphization doses $\left(\Phi_{\mathrm{am}}\right)$ for ions of medium energy with a mass of $M_{1} \leq 31$ a.m.u. were calculated. It is assumed that amorphization at a given depth occurs at a dose at which a certain threshold value of the total concentration of vacancies and divacancies $\left(C_{\mathrm{am}}\right)$ is reached. Variable parameters in the calculations were: ion energy, ion current density, temperature, as well as the threshold atomic displacement energy of atom $\left(E_{d}\right)$ and $C_{\text {am. }}$. Limits of the diffusion-coagulation model are determined. Comparison of the results of calculations carried out at these limits with published experimental data showed (taking into account the variation of the experimental data and a certain freedom of choice of the parameters $E_{d}$ and $C_{\text {am }}$ ) a satisfactory agreement between the calculated and experimental values of $\Phi_{\mathrm{am}}$.
\end{abstract}

